\title{
Estratégias para lidar com as ações judiciais de medicamentos no estado de São Paulo
}

\author{
Strategies to deal with drug lawsuits in the state of São Paulo, Brazil
}

Las estrategias para hacer frente a las demandas de las drogas en el estado de Sao Paulo, Brasil

Tereza Setsuko Toma ${ }^{1}$

Amanda Cristiane Soares ${ }^{2}$

Paula Sue Facundo de Siqueira ${ }^{3}$

Robert Domingues ${ }^{4}$

RESUMO: O Sistema Único de Saúde (SUS) apresentou muito avanços desde sua criação há quase trinta anos, no entanto, ainda enfrenta grandes desafios. Dificuldades persistentes na oferta de atenção integral à saúde podem ter contribuído para a utilização da via judicial para o acesso a medicamentos. $\mathrm{O}$ fenômeno da judicialização da saúde passou a ter maior visibilidade no estado de São Paulo no início dos anos 2000 a partir da publicação de resultados de algumas pesquisas. Após 2005, o volume crescente de ações judiciais para obtenção de medicamentos e seus custos exorbitantes levaram à implementação de diversas estratégias por parte da Secretaria de Estado da Saúde de São Paulo e de instâncias do sistema judiciário para lidar com o problema. Alguns resultados foram alcançados, porém ainda são insuficientes, uma vez que continuam ocorrendo ações para medicamentos que não têm sua eficácia e segurança comprovados. Este artigo apresenta as estratégias adotadas no estado de São Paulo para lidar com as ações judiciais para obtenção de medicamentos.

Palavras-chave: Decisões judiciais. Direito sanitário. Assistência farmacêutica.

ABSTRACT: The Brazilian Unified Health System (SUS) has made great advances since its creation almost thirty years ago, but it still faces great challenges. Persistent difficulties in providing comprehensive health care may have contributed to the use of the judicial pathway for access to medicines. The phenomenon of health judicialization began to have greater visibility in the state of São Paulo in the early 2000s from the publication of results of some research. After 2005, the increasing volume of lawsuits to obtain medicines and their exorbitant costs led to the implementation of several strategies by the State Department of Health of São Paulo and judicial system instances to deal with the problem. Some results

\footnotetext{
${ }^{1}$ Médica. Pesquisadora científica VI. Doutora em Nutrição em Saúde Pública. Diretora do Centro de Tecnologias de Saúde para o SUS-SP do Instituto de Saúde da Secretaria de Estado da Saúde de São Paulo. São Paulo, SP, Brasil. E-mail: ttoma@isaude.sp.gov.br

2 Tecnóloga em saúde. Especialista em Economia e Avaliação de Tecnologias em Saúde. Diretora do Núcleo de Análise e Projetos de Avaliação de Tecnologias de Saúde do Instituto de Saúde da Secretaria de Estado da Saúde de São Paulo. São Paulo, SP, Brasil. E-mail: amanda.soares@isaude.sp.gov.br

${ }^{3}$ Especialista em Administração Pública. Especialista em Direito Sanitário. Coordenadora das demandas judiciais em saúde do estado de São Paulo. Secretaria de Estado da Saúde de São Paulo. Coordenação de Demandas Estratégicas do SUS, São Paulo, SP, Brasil. E-mail: psue@saude.sp.gov.br

${ }_{4}^{4}$ Administrador de empresas, graduando em Farmácia. Assistente técnico III, Analista de ações judiciais em saúde. Coordenação de Demandas Estratégicas do SUS, São Paulo, SP, Brasil. E-mail: robertdomingues@saude.sp.gov.br
} 
have been achieved, but they are still insufficient, since actions for drugs that do not have their proven efficacy and safety continue to occur. This article presents the strategies adopted in the state of São Paulo to deal with lawsuits to obtain drugs.

Keywords: Judicial decisions. Health law. Pharmaceutical services.

RESUMEN: El Sistema de Salud de Brasil (SUS) mostró mucho progreso desde su creación hace casi treinta años, sin embargo, todavía se enfrenta a grandes retos. Las dificultades persistentes para ofrecer una atención integral de la salud pueden haber contribuido a la utilización de los tribunales para el acceso a los medicamentos. La legalización del fenómeno de la salud tiene una mayor visibilidad en el estado de Sao Paulo en la década de 2000 después de la publicación de los resultados de algunas investigaciones. Después de 2005, el número creciente de demandas para la obtención de medicamentos y sus costos exorbitantes llevó a la implementación de diversas estrategias por parte de la Secretaría de Salud de Sao Paulo, y las instancias del sistema judicial para lidiar con el problema. Se lograron algunos resultados, pero aún son insuficientes, ya que las acciones se siguen produciendo por los medicamentos que no han demostrado su eficacia y seguridad. Este artículo presenta las estrategias adoptadas en el estado de Sao Paulo para hacer frente a las demandas para obtener medicamentos.

Palabras-Ilave: Decisiones judiciales. Derecho sanitario. Servicios farmacéuticos.

\section{Introdução}

O direito de atenção à saúde, garantido pela Constituição Federal de 1988, levou à instituição do Sistema Único de Saúde - SUS, pautado pelos princípios de universalidade, integralidade, descentralização e participação social. Uma análise realizada por Piola e colaboradores sobre o SUS, vinte anos após a Constituição (1), indicou que muitos avanços foram alcançados:

Ao longo dessas duas décadas, ainda que enfrentando dificuldades de financiamento e resistências de ordem política e cultural, o processo de implantação do sistema público de saúde de acesso universal, que se propôs a oferecer cobertura integral às necessidades da população, colecionou muitos avanços. Um arrojado processo de descentralização expandiu significativamente a participação das esferas subnacionais na gestão e no financiamento do sistema, bem como na provisão de ações e serviços de saúde, resultando na redução das desigualdades interregionais que prevaleciam no sistema pré-Constituição Federal de 1988.

Diretrizes, políticas e programas inovadores introduziram importantes mudanças no modelo de atenção à saúde e ampliaram o espectro de ações e serviços ofertados, assegurando a um número crescente de cidadãos o acesso a recursos terapêuticos antes indisponíveis. 
O SUS, no entanto, ainda enfrenta grandes desafios para garantir a integralidade da atenção à saúde e o acesso oportuno da população a determinados serviços. Essas dificuldades persistentes explicariam em parte a tendência de parcela da população de recorrer à via judicial, fenômeno conhecido como judicialização da saúde. Desta forma, uma visão do problema é que o Judiciário, ao assegurar os direitos constitucionais, estaria procurando corrigir essas deficiências. Um exemplo citado é o caso de dispensa de medicamentos para tratamento de Aids, em que a pressão de um grupo pequeno por via judicial levou ao desenvolvimento de política pública específica para acesso ao tratamento integral. De outro lado, a visão defendida por gestores públicos é que o uso da via judicial tem gerado distorções no sistema, uma vez que os recursos financeiros são escassos (1). Destaca-se a discussão em torno de um conflito distributivo causado por essa forma de acesso a direitos, tais como a priorização do direito individual sobre o coletivo e a possibilidade de favorecimento das pessoas financeiramente mais privilegiadas que teriam condições de arcar com os custos judiciais (1).

No início dos anos 2000 a judicialização da saúde passou a ter visibilidade no estado de São Paulo em função de vários estudos que analisaram as ações judiciais para obtenção de medicamentos.

Um estudo foi realizado por Marques e Dallari (2)., em 2004, com o objetivo de verificar como o sistema jurídico atuava para garantir o direito social à assistência farmacêutica. Os 31 processos selecionados nas Varas da Fazenda Pública do Estado de São Paulo, e datados de 1997 a 2004, foram analisados com relação aos seus discursos. Constatou-se que os autores dos processos eram indivíduos representados por advogados particulares na maioria das vezes $(67,7 \%)$, e vários com apoio de associações $(23,8 \%)$. Os réus nos processos com grande frequência eram o Secretário de Estado da Saúde (61\%) e a Fazenda Pública (22\%). Em 93,5\% das decisões judiciais analisadas foram concedidas liminares para que o Estado fornecesse o medicamento solicitado nas ações. E em 90,3\% das sentenças proferidas por juízes as demandas foram julgadas como procedentes. As autoras concluíram que o Poder Judiciário ao adotar essa conduta não levava em consideração a política pública de medicamentos, ignorando desta forma que os direitos foram instituídos atrelados a políticas sociais e econômicas. Além do mais, as ações judiciais poderiam acobertar interesses de determinados laboratórios farmacêuticos 
Vieira e Zucchi (3), a partir de análise documental, estudaram as ações judiciais para obtenção de medicamentos movidas contra a Secretaria Municipal da Saúde de São Paulo, durante $o$ ano de 2005. Verificou-se que foram impetradas 170 ações, que implicaram em recurso extra de 876 mil reais para compra dos medicamentos solicitados. A maioria das ações foi demandada por mulheres, e por pessoas com menos de 19 anos e idosos. As doenças mais referidas nessas ações foram diabetes, câncer, hipertensão arterial, osteoporose e hepatite. Constatou-se que 59\% das prescrições eram oriundas do SUS e que $62 \%$ dos medicamentos solicitados faziam parte de listas do SUS. Essas ações foram movidas em $54 \%$ das vezes por advogados particulares, e frequentemente (63\%) por cidadãos residentes em áreas com menor grau de exclusão social. Levantaram-se algumas hipóteses para explicar esses achados: as mulheres teriam maior consciência sobre saúde, jovens e idosos apresentariam doenças mais graves e de longa duração, doenças crônicas teriam custo mais elevado o que justificaria o investimento em contratar um advogado, o sistema público poderia ter falhado na garantia de acesso a medicamentos, o prescritor desconhecia a disponibilidade de medicamentos no SUS. As autoras argumentaram que estas ações criavam dificuldades para a gestão do SUS e indicaram a necessidade de se encontrar uma solução partilhada entre o sistema judiciário e o executivo

Outro estudo, conduzido por Chieffi e Barata (5), analisou 2.927 ações judiciais cadastradas durante o ano de 2006 no sistema de registro eletrônico das demandas jurídicas da Secretaria de Estado da Saúde de São Paulo (SES-SP), e movidas por moradores da capital do estado. De 954 itens de medicamentos solicitados, 77\% não faziam parte dos programas de assistência farmacêutica, e $3 \%$ não estavam disponíveis no mercado nacional. Verificou-se que $17 \%$ dos medicamentos eram relativos ao aparelho digestivo e metabolismo, $17 \%$ ao sistema cardiovascular e $16 \%$ ao sistema nervoso. Com relação às receitas médicas, $48 \%$ eram provenientes de serviços do SUS e $47 \%$ do sistema suplementar. A maioria dos pacientes demandantes (74\%) residia em áreas de menor vulnerabilidade social. As autoras concluíram que ao desconsiderar a dimensão coletiva, os processos judiciais contribuíam para reforçar as desigualdades sociais na atenção à saúde (4). Em 2006, calculou-se que o gasto foi de 18 mil reais por paciente de ação judicial, um valor exorbitante se comparado com aos 2,2 mil reais por paciente efetuado pelo Programa 
de Medicamentos de Dispensação Excepcional. Além disso, chamava à atenção a concentração de processos por advogado.

O volume crescente de demandas judiciais para obtenção de medicamentos levou ao desenvolvimento de várias iniciativas no estado de São Paulo para lidar com o problema: Coordenação das Demandas Estratégicas do SUS, Procuradoria Geral do Estado, atendimento administrativo da SES-SP, Projeto SUS do Tribunal de Justiça do Estado de São Paulo, Triagem Farmacêutica no Juizado Especial da Fazenda Pública, Rede Paulista de Avaliação de Tecnologias em Saúde, Comissão de Farmacologia.

O objetivo deste artigo é relatar as estratégias adotadas pelas instituições mencionadas ao longo dos anos e os resultados alcançados.

\section{Coordenação das Demandas Estratégicas do SUS}

A Coordenação das Demandas Estratégicas do SUS - CODES, uma instância informal vinculada ao Gabinete do Secretário de Saúde da SES-SP, desenvolveu em 2005 uma ferramenta informatizada para apoio à gestão denominada Sistema de Controle Jurídico. Esse sistema foi aprimorado em 2009 para apoiar também as áreas responsáveis pela aquisição e dispensa de medicamentos e materiais. O novo sistema, S-CODES, foi implantado em todo o estado em 2010, permitindo o cadastramento das demandas judiciais de todos os Departamentos Regionais de Saúde - DRS (6).

\section{Procuradoria Geral do Estado}

As ações judiciais no Estado de São Paulo tomaram forma e proporção para o setor judiciário quando a Procuradora Maria Luciana de Oliveira Facchina Podval analisou os processos que compunham a sua banca na Procuradoria Judicial entre os anos de $2001 \mathrm{e}$ 2008. O resultado foi extremamente relevante, visto que se constatou que as ações judiciais de saúde contra o Estado cresceram expressivamente, ano a ano, passando de 17\%, em 2001, para $85 \%$ de todas as ações ao final da análise. A partir desse ponto, era mais do que necessário adotar uma atitude para tentar controlar a situação (7).

Pensando nesse enfrentamento, a Procuradoria Geral do Estado - PGE criou pela primeira vez, em 2007, uma área especializada em saúde pública, a $8^{\text {a }}$ Subprocuradoria da Procuradoria Judicial - PJ8. Como competência, a PJ8 trabalha com ações que tenham 
como objeto o fornecimento de medicamentos pelo Estado, bem como as demandas que digam respeito às políticas públicas na área da saúde 5 .

A partir de então foi estabelecida uma parceria entre a PGE e a SES-SP que trouxe grandes avanços em resposta ao fenômeno da judicialização. O projeto "Uma parceria inovadora para fazer frente ao fenômeno da judicialização das políticas públicas de saúde a otimização da gestão pública" recebeu o Prêmio Mário Covas de 2008, na categoria Inovação em Gestão Pública (8).

Em 2009, o Procurador Geral do Estado, considerando a necessidade de uma coordenação para esse trabalho complexo e bastante técnico, instituiu a Coordenadoria Judicial de Saúde Pública - COJUSP no âmbito da PJ8. Dentre suas diversas atribuições inclui-se a orientação e apoio técnico aos Procuradores do Estado, a fim de harmonizar as condutas e institucionalizar canais de comunicação, sempre visando a aproximação do órgão jurídico e de saúde do estado (8).

\section{Atendimento administrativo}

Em 2009, a SES-SP lançou uma via de solicitação de medicamentos, chamada de atendimento administrativo (6). Após uma triagem, na impossibilidade de solucionar o problema do paciente demandante por meio de sua reinserção no SUS ou oferta de alternativas terapêuticas, uma equipe multidisciplinar da SES-SP avalia a solicitação do médico e emite, quando apropriado, uma autorização para fornecimento excepcional. A solicitação é feita por meio de uma série de documentos, tais como documento de identificação do paciente, relatório, receita médica e formulário específico (8).

No atendimento administrativo, o gestor pode padronizar os itens dispensados, o que contribui para o poder de negociação na compra dos itens e sua entrega (8).

Outro passo importante tomado pela SES-SP foi com relação ao fornecimento de medicamentos oncológicos, comprovadamente considerados imprescindíveis, no Centros de Alta Complexidade em Oncologia - CACON e Unidades de Assistência de Alta Complexidade em Oncologia - UNACON. Apesar do avanço nos últimos anos na

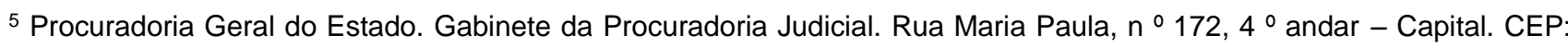
01319-000. REF.: MAPEAMENTO CONTENCIOSO. 30 p. Disponível em: http://apesp.org.br/wpcontent/uploads/2015/10/mapeamento_procuradoria_judicial310715-12.pdf
} 
incorporação de alguns desses medicamentos pela Relação Nacional de Medicamentos RENAME, o estado de SP ainda cobre parte dos não incorporados (8).

\section{Projeto SUS do Tribunal de Justiça do Estado de São Paulo}

Em resposta à Recomendação do Conselho Nacional de Justiça oㅜ 31, que "Recomenda aos Tribunais a adoção de medidas visando melhor subsidiar os magistrados e demais operadores do direito, para assegurar maior eficiência na solução das demandas judiciais envolvendo a assistência à saúde" (9), em 2012 foi assinado o Termo de Cooperação Técnica que celebrou um convênio entre Governo do Estado, Tribunal de Justiça do Estado de São Paulo - TJSP e Escola Paulista de Magistratura - EPM6.

Dessa forma, iniciou-se o "Projeto SUS no TJSP", que consiste na disponibilização de ferramentas de apoio e informações técnicas para subsidiar as decisões dos magistrados de maneira rápida e prática. No site da EPM foi disponibilizado um banco de dados de medicamentos e um canal direto com pesquisadores selecionados pela Secretaria de Estado da Saúde, que devem responder em até 72 horas (8).

\section{Triagem Farmacêutica no Juizado Especial da Fazenda Pública}

Em 2013, ainda em resposta à Recomendação do Conselho Nacional de Justiça no 31, foi criada a Triagem Farmacêutica no Juizado Especial da Fazenda Pública - JEFAZ (8). O projeto JEFAZ, premiado com menção honrosa do Instituto Innovare, tem como proposta fazer uma análise prévia do processo, por técnicos da SES-SP junto às varas dos juizados especiais da Fazenda Pública, a fim de identificar outros meios para o paciente, que não seja a judicialização. Quando pertinente, são dadas orientações sobre os Programas Oficiais de Assistência Farmacêutica do SUS e seus Protocolos, a fim de reinserir o paciente no SUS, quando possível (8).

6 Saúde firma termo de cooperação com TJ-SP e Escola Paulista de Magistratura. Disponível em: http://www.saude.sp.gov.br/ses/noticias/2012/setembro/saude-firma-termo-de-cooperacao-com-tj-sp-e-escola-paulista-demagistratura. 


\section{Rede Paulista de Avaliação de Tecnologias em Saúde}

A Rede Paulista de Avaliação de Tecnologias em Saúde - REPATS, formada pelos Núcleos de Avaliação de Tecnologia em Saúde - NATS presentes em hospitais e instituições de ensino e assistência à saúde, funciona desde 2011 como uma rede de colaboração dentro do Estado de São Paulo7. Aos NATS cabe articular um conjunto de ações de ATS, solucionando problemas e atendendo às suas demandas, fazendo uso de instrumentos consagrados como avaliações da eficácia, efetividade, eficiência e segurança das intervenções de diagnóstico, prevenção, tratamento, recuperação ou cuidados paliativos, e também a criação de diretrizes terapêuticas baseada em evidências, visando sempre o uso racional de tecnologias e principalmente a segurança do paciente (10).

A REPATS realiza reuniões mensais, em que são discutidas suas produções e temas de relevância para a área de ATS. Vista como uma forma de motivação para a rede se movimentar, compartilhar experiências e estabelecer parcerias, essas reuniões atualmente são transmitidas ao vivo e gravadas pela Unidade de Telemedicina do Instituto do Coração da Faculdade de Medicina da Universidade de São Paulo, via parceria da Rede Universitária de Telemedicina - RUTE. Outra iniciativa da REPATS com o objetivo de impulsionar a criação de novos NATS e fortalecer os já existentes, é a formação de capital humano por meio de oficinas de capacitação em ATS realizadas mensalmente (11).

\section{Comissão de Farmacologia}

O atendimento administrativo foi regulamentação em 2012, com a aprovação da estrutura e funcionamento da Comissão de Farmacologia da SES-SP, em consonância com a Política Nacional de Medicamentos, e com o objetivo de assessorar na formulação de diretrizes para seleção, padronização, prescrição, aquisição, distribuição, dispensação e seguimento farmacoterapêutico no âmbito do Sistema Único de Saúde do Estado de São Paulo. Desta forma, a solicitação de medicamento por protocolo clínico de tratamento de instituição pública de saúde passou a ser realizado de acordo com formulário específico de solicitação de medicamento, para posterior análise da comissão. A solicitação de medicamento por paciente em caráter de excepcionalidade pode ser realizada, desde que

\footnotetext{
${ }^{7}$ Núcleos de Avaliação de Tecnologias em Saúde (NATS). Disponível em: http://ses.sp.bvs.br/vhl/coordenadoria-deciencia-tecnologia-e-inovacao-em-saude/nucleos-de-avaliacao-de-tecnologias-em-saude-nats/
} 
esgotadas todas as alternativas terapêuticas disponibilizadas pelo SUS, e com base em forte nível de evidência científica para sua utilização (12).

\section{Evolução das ações judiciais de 2010 a 2015}

Com a finalidade de verificar os resultados das iniciativas adotadas no estado de São Paulo para lidar com o fenômeno da judicialização da saúde, alguns dados sobre demandas de ações judiciais de medicamentos nos anos de 2010 a 2015 foram extraídos do S-CODES.

Os dados indicam que apesar das estratégias postas em prática, tanto pela SES, quanto pelo sistema judiciário, as demandas de medicamentos por meio de ações judiciais vêm aumentando ano a ano, sendo que em 2015 o número foi quase o dobro de 2010, respectivamente 18.045 e 9.385. Quanto ao atendimento administrativo, triplicou o número de demandas de 2010 a 2013 (de 7.688 para 22.675), seguido de uma redução em torno de 25\% em 2015 (figura 1).

Figura 1. Número de demandas de medicamentos cadastradas ano a ano, por meio de ações judiciais e atendimento administrativo, no estado de São Paulo de 2010 a 2015.

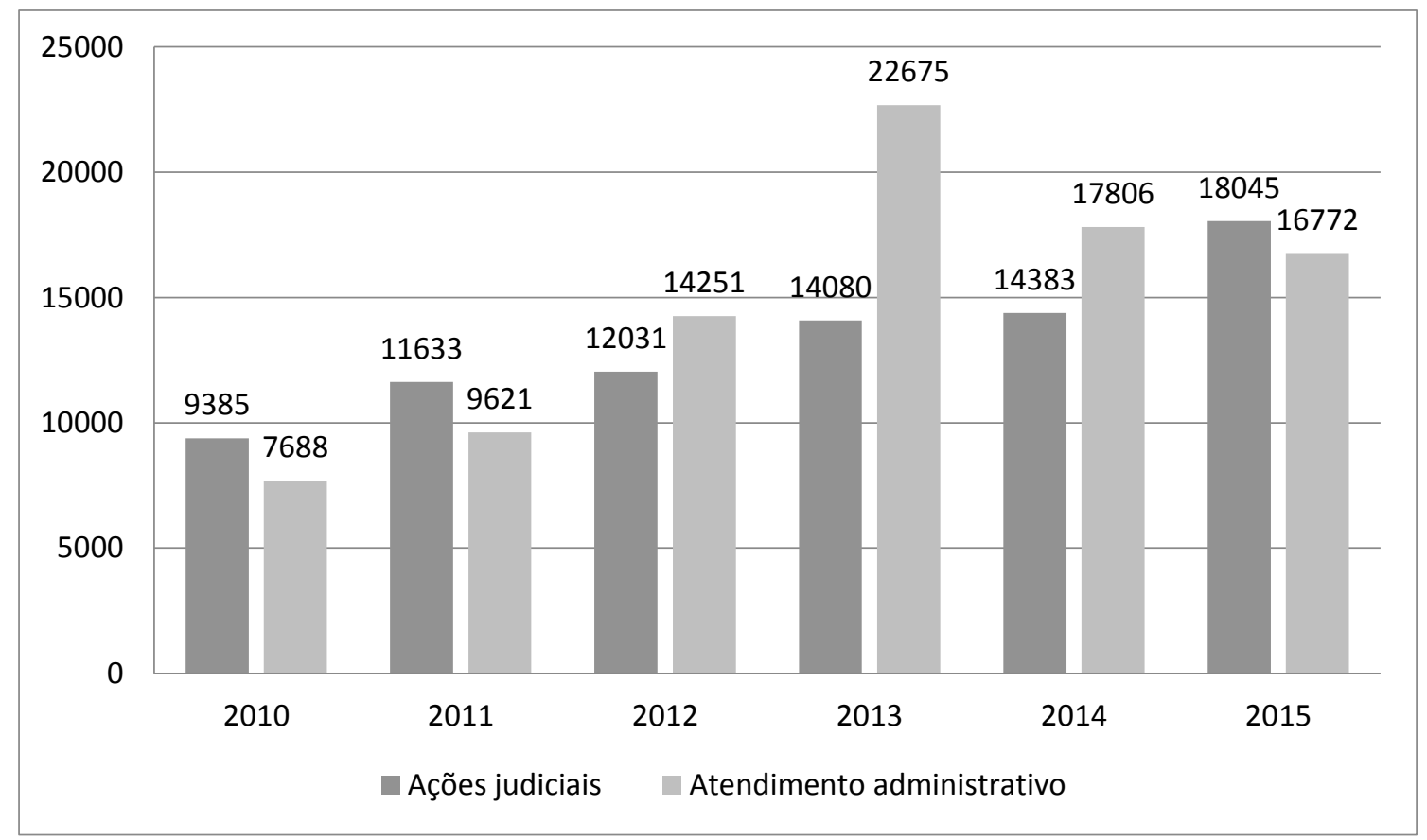

Fonte: Elaborada pelos autores a partir de informações extraídas do S-Codes. 
O Índice Paulista de Judicialização em Saúde - IPJS, calculado como o número de ações em relação a 10.000 habitantes, mostra que o fenômeno da judicialização para obter medicamentos continua em crescimento no estado de São Paulo, e é heterogêneo entre os dezessete Departamentos Regionais de Saúde da SES-SP, conforme o quadro 1.

Em 2015, observa-se que o IPJS variou de 0,25 (DRS 12 - Registro) a 26,41 (DRS 5 - Barretos), e que dez DRS apresentaram índices acima da média estadual. Chama a atenção os índices bastante elevados nas regiões correspondentes aos DRS Barretos, Franca, Marília, Presidente Prudente, Ribeirão Preto e São José do Rio Preto. 
Quadro 1. Índice Paulista de Judicialização em Saúde (IPJS) de novas ações para obtenção de medicamentos, conforme as dezessete regionais de saúde da Secretaria de Estado da Saúde de São Paulo, nos anos de 2011 e 2015.

\begin{tabular}{|c|c|c|c|c|c|c|c|c|c|c|c|c|c|c|}
\hline \multirow{2}{*}{ Regional de Saúde } & \multicolumn{3}{|c|}{2011} & \multicolumn{3}{|c|}{2012} & \multicolumn{3}{|c|}{2013} & \multicolumn{3}{|c|}{2014} & \multicolumn{2}{|r|}{2015} \\
\hline & $\begin{array}{c}\text { Ações } \\
\text { Judiciais }\end{array}$ & População & Índice & \begin{tabular}{|c|} 
Ações \\
Judiciais
\end{tabular} & População & Índice & $\begin{array}{l}\text { Aç̃ões } \\
\text { Judiciais }\end{array}$ & População & Índice & $\begin{array}{c}\text { Aç̃̃es } \\
\text { Judiciais }\end{array}$ & População & Índice & $\begin{array}{c}\text { Ações } \\
\text { Judiciais }\end{array}$ & População İndice \\
\hline DRS 01 - Grande São Paulo & 1.281 & 19.867 .456 & 0,64 & 1.402 & 19.956 .590 & 0,70 & 1.389 & 20.128 .227 & 0,69 & 1.274 & 20.284 .891 & 0,63 & 1.508 & $21.090 .791 \quad 0,72$ \\
\hline DRS 02 - Araçatuba & 335 & 725.553 & 4,62 & 235 & 728.743 & 3,22 & 342 & 733.528 & 4,66 & 375 & 738.544 & 5,08 & 412 & $769.174 \quad 5,36$ \\
\hline DRS 03 - Araraquara & 318 & 929.823 & 3,42 & 323 & 934.863 & 3,46 & 381 & 943.045 & 4,04 & 395 & 951.086 & 4,15 & 521 & $991.129 \quad 5,26$ \\
\hline DRS 04 - Baixada Santista & 195 & 1.683 .725 & 1,16 & 439 & 1.692 .425 & 2,59 & 320 & 1.713 .741 & 1,87 & 203 & 1.731 .403 & 1,17 & 211 & $1.797 .500 \quad 1,17$ \\
\hline DRS 05 - Barretos & 641 & 414.030 & 15,48 & 795 & 412.878 & 19,26 & 834 & 417.471 & 19,98 & 1.091 & 419.161 & 26,03 & 1.143 & $432.83026,41$ \\
\hline DRS 06 - Bauru & 705 & 1.640 .540 & 4,30 & 619 & 1.648 .443 & 3,76 & 709 & 1.660 .609 & 4,27 & 617 & 1.673 .337 & 3,69 & 779 & $1.744 .292 \quad 4,47$ \\
\hline DRS 07 - Campinas & 418 & 4.098 .799 & 1,02 & 457 & 4.128 .965 & 1,11 & 498 & 4.201.231 & 1,19 & 513 & 4.261 .670 & 1,20 & 502 & $4.433 .543 \quad 1,13$ \\
\hline DRS 08 - Franca & 548 & 655.726 & 8,36 & 342 & 658.943 & 5,19 & 443 & 664.301 & 6,67 & 456 & 669.387 & 6,81 & 574 & $696.600 \quad 8,24$ \\
\hline DRS 09 - Marília & 375 & 1.074 .333 & 3,49 & 278 & 1.077 .491 & 2,58 & 316 & 1.080 .783 & 2,92 & 345 & 1.085 .161 & 3,18 & 1.289 & $1.128 .94111,42$ \\
\hline DRS 10 - Piracicaba & 143 & 1.428 .857 & 1,00 & 234 & 1.437 .195 & 1,63 & 226 & 1.451 .810 & 1,56 & 201 & 1.465 .675 & 1,37 & 228 & $1.527 .411 \quad 1,49$ \\
\hline DRS 11 - Presidente Prudente & 330 & 726.081 & 4,54 & 436 & 728.122 & 5,99 & 468 & 731.355 & 6,40 & 566 & 734.571 & 7,71 & 586 & $762.528 \quad 7,68$ \\
\hline DRS 12 - Registro & 5 & 273.982 & 0,18 & 9 & 274.064 & 0,33 & 7 & 274.308 & 0,26 & 6 & 274.581 & 0,22 & 7 & $284.031 \quad 0,25$ \\
\hline DRS 13 - Ribeirão Preto & 1.052 & 1.347 .602 & 7,81 & 909 & 1.357 .165 & 6,70 & 1.187 & 1.375 .297 & 8,63 & 1.414 & 1.392 .126 & 10,16 & 1.357 & $1.452 .570 \quad 9,34$ \\
\hline DRS 14 - São João da Boa Vista & 457 & 778.353 & 5,87 & 312 & 780.842 & 4,00 & 260 & 785.056 & 3,31 & 261 & 789.006 & 3,31 & 404 & $818.983 \quad 4,93$ \\
\hline DRS 15 - São José do Rio Preto & 1.234 & 1.484 .270 & 8,31 & 1.209 & 1.493 .835 & 8,09 & 1.201 & 1.501 .730 & 8,00 & 1.376 & 1.512 .857 & 9,10 & 1.402 & $1.579 .7158,88$ \\
\hline DRS 16 - Sorocaba & 159 & 2.271 .483 & 0,70 & 200 & 2.284 .897 & 0,88 & 333 & 2.308 .173 & 1,44 & 525 & 2.331 .330 & 2,25 & 633 & $2.433 .0592,60$ \\
\hline DRS 17 - Taubaté & 183 & 2.292 .055 & 0,80 & 209 & 2.305 .758 & 0,91 & 192 & 2.334 .029 & 0,82 & 161 & 2.358 .600 & 0,68 & 226 & $2.453 .387 \quad 0,92$ \\
\hline Total Geral & 8.379 & 41.692 .668 & 2,01 & 8.408 & 41.901 .219 & 2,01 & 9.106 & 42.304 .694 & 2,15 & 9.779 & 42.673 .386 & 2,29 & 11.782 & $44.396 .484 \quad 2,65$ \\
\hline $\begin{array}{l}000 \text { habitantes } \\
\text { ão Fundação Seade }\end{array}$ & 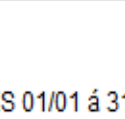 & 12015 & & & & & & & & & & & & \\
\hline
\end{tabular}

Fonte: Elaborado pelos autores a partir de informações extraídas do S-Codes. 
Quadro 2. Contribuição dos seis Departamentos Regionais de Saúde (DRS) com Índice Paulista de Judicialização em Saúde mais elevados, nos dez medicamentos mais demandados, de 2011 a 2015 no estado de São Paulo.

\begin{tabular}{|c|c|c|c|c|c|c|c|c|}
\hline \multirow[b]{2}{*}{$\begin{array}{l}\text { Descrição dos } 10 \text { itens mais demandados } \\
\text { (princípio ativo) }\end{array}$} & \multicolumn{7}{|c|}{ Número de ações (\% em relação ao estado de São Paulo) } & \multirow[b]{2}{*}{$\begin{array}{l}\text { Estado de } \\
\text { São Paulo }\end{array}$} \\
\hline & $\begin{array}{l}\text { DRS } 5 \\
\text { Barretos }\end{array}$ & $\begin{array}{l}\text { DRS } 8 \\
\text { Franca }\end{array}$ & $\begin{array}{l}\text { DRS9 } \\
\text { Marília }\end{array}$ & $\begin{array}{l}\text { DRS } 11 \\
\text { Presidente } \\
\text { Prudente }\end{array}$ & $\begin{array}{c}\text { DRS 13 } \\
\text { Ribeirão } \\
\text { Preto }\end{array}$ & $\begin{array}{c}\text { DRS } 15 \\
\text { São José do } \\
\text { Rio Preto }\end{array}$ & $\begin{array}{l}\text { Total dos } 6 \\
\text { DRS }\end{array}$ & \\
\hline Insulina glargina - $100 \mathrm{Ui} / \mathrm{Ml}$ - $3 \mathrm{Ml}$ - Refil / Unidade & $85(3,0)$ & $15(0,5)$ & $465(16,7)$ & $52(1,9)$ & $129(4,6)$ & $268(9,6)$ & $1.014(36,4)$ & 2.783 \\
\hline Ranibizumabe - $10 \mathrm{Mg} / \mathrm{Ml}-0,23 \mathrm{Ml} /$ Ampola & $130(7,2)$ & $136(7,5)$ & $6(0,0)$ & $204(11,3)$ & $263(14,6)$ & $341(18,9)$ & $1.080(59,8)$ & 1.807 \\
\hline Insulina lispro - $100 \mathrm{Ui} / \mathrm{Ml}$ - $3 \mathrm{Ml}$ - Refil / Unidade & - & $8(0,5)$ & $531(35,7)$ & $19(1,28)$ & $40(2,69)$ & $39(2,62)$ & $637(42,8)$ & 1.489 \\
\hline Clopidogrel - 75 Mg / Comprimido & $174(14,7)$ & $119(10,1)$ & $38(3,2)$ & $50(4,2)$ & $193(16,3)$ & $145(12,3)$ & $719(60,8)$ & 1.182 \\
\hline Insulina asparte - 100 Ui/Ml - 3 Ml - Refil / Unidade & - & - & $193(17,3)$ & $5(0,4)$ & $45(4,0)$ & - & $243(21,8)$ & 1.116 \\
\hline $\begin{array}{l}\text { Cloridrato de Cinacalcete - } 30 \mathrm{Mg} \text { / Comprimido } \\
\text { Revestido }\end{array}$ & $28(3,0)$ & $73(7,7)$ & - & $14(1,5)$ & $186(19,7)$ & $75(7,9)$ & $376(39,8)$ & 945 \\
\hline $\begin{array}{l}\text { Hialuronato de Sódio - } 10 \text { Mg/Ml - Solução Injetável - } 2 \\
\text { Ml / Frasco-Ampola }\end{array}$ & $185(24,5)$ & - & $5(0,7)$ & - & - & $119(15,7)$ & $309(40,9)$ & 756 \\
\hline Boceprevir - 200 Mg / Cápsula Gelatinosa & - & - & - & $19(2,6)$ & - & - & $19(2,6)$ & 737 \\
\hline Glicosamina + Condroitina - 1,5 G + 1,2 G / Sache & $81(11,4)$ & - & $22(3,1)$ & $7(1,0)$ & $54(7,6)$ & $38(5,3)$ & $202(28,4)$ & 711 \\
\hline Rivaroxabana - $20 \mathrm{Mg}$ / Comprimido Revestido & $82(11,9)$ & $70(10,1)$ & $28(4,1)$ & $8(1,2)$ & $145(21,0)$ & $73(10,6)$ & $479(69,4)$ & 690 \\
\hline Total & $765(6,3)$ & $421(3,4)$ & $1.288(10,5)$ & $378(3,1)$ & $1.055(8,6)$ & $1.098(9,0)$ & $5.078(41,6)$ & 12.216 \\
\hline
\end{tabular}

Fonte: Elaborado pelos autores a partir de informações extraídas do S-Codes. 
No quadro 2 estão os dez medicamentos mais demandados por ações judiciais de 2011 a 2015. Seis desses medicamentos foram analisados pela Comissão Nacional de Incorporação de Tecnologias no SUS - CONITEC, porém não foram incorporados: insulinas glargina, lispro e asparte para diabetes mellitus (13), (14); ranizumabe foi avaliado para degeneração macular relacionada à idade (15), mas não para edema macular diabético e edema macular secundário à oclusão de veia da retina (16), (17); rivaroxabana para prevenção de acidente vascular cerebral (18); hialuronato de sódio para dor associada com osteoartrose do joelho (19). Glicosamina + condroitina para osteoartrite não foi avaliado pela CONITEC, mas há uma nota técnica do NATS da Universidade Federal de Minas Gerais indicando não haver efeito comprovado de benefícios (20). Boceprevir foi incorporado no SUS em 2012 para tratar hepatite C, e excluído em 2016, devido à incorporação de outros medicamentos mais apropriados (21), (22). Cloridrato de cinacalcete foi incorporado em 2015 para hiperparatireoidismo secundário à doença renal, em diálise e refratários à terapia convencional (23), (24). Clopidogrel faz parte dos componentes especializado e hospitalar da Rename (25). 
Quadro 3. Situação dos dez medicamentos mais demandados por ações judiciais de 2011 a 2015 no Estado de São Paulo quanto à decisão por incorporação no SUS.

\begin{tabular}{|c|c|c|}
\hline Descrição do Item (princípio ativo) & Indicação & Decisão quanto à incorporação no SUS \\
\hline $\begin{array}{l}\text { Boceprevir - } 200 \text { mg / cápsula } \\
\text { gelatinosa }\end{array}$ & Hepatite C & $\begin{array}{l}\text { Avaliado pela CONITEC. Decisão de incorporar ao SUS publicada em 26/07/2012. } \\
\text { Avaliado pela CONITEC. Decisão de excluir do SUS publicada em 24/05/2016. }\end{array}$ \\
\hline Clopidogrel - $75 \mathrm{mg} /$ comprimido & Síndrome coronariana aguda & Faz parte da RENAME - Componente especializado e procedimento hospitalar - B01AC04 \\
\hline $\begin{array}{l}\text { Cloridrato de cinacalcete - } 30 \mathrm{mg} / \\
\text { comprimido revestido }\end{array}$ & $\begin{array}{l}\text { Hiperparatireoidismo secundário à } \\
\text { doença renal, em diálise e refratários } \\
\text { à terapia convencional }\end{array}$ & $\begin{array}{l}\text { Avaliado pela CONITEC. Decisão de não incorporar ao SUS publicada em 15/10/2013. } \\
\text { Avaliado pela CONITEC. Decisão de incorporar ao SUS publicada em 30/09/2015. }\end{array}$ \\
\hline $\begin{array}{l}\text { Glicosamina + Condroitina - } 1,5 \mathrm{~g}+ \\
1,2 \mathrm{~g} / \mathrm{sache}\end{array}$ & Osteoartrite & $\begin{array}{l}\text { Não avaliado pela CONITEC. } \\
\text { Avaliado pelo NATS/UFMG em 2013: O sulfato de glicosamina e o sulfato de condroitina não } \\
\text { tem efeito comprovado no controle da dor e na regeneração da cartilagem articular nos casos } \\
\text { de osteoartrite. }\end{array}$ \\
\hline $\begin{array}{l}\text { Hialuronato de sódio - } 10 \text { mg/ml - } \\
\text { solução injetável - } 2 \mathrm{ml} \text { / frasco- } \\
\text { ampola }\end{array}$ & $\begin{array}{l}\text { Dor associada com a osteoartrose } \\
\text { do joelho. }\end{array}$ & Avaliado pela CONITEC. Decisão de não incorporar ao SUS publicada em 17/12/2014. \\
\hline $\begin{array}{l}\text { Insulina asparte - } 100 \mathrm{Ui} / \mathrm{ml}-3 \mathrm{ml}- \\
\text { refil / unidade }\end{array}$ & Diabetes mellitus & Avaliado pela CONITEC. Decisão de não incorporar ao SUS publicada em 05/09/2014. \\
\hline $\begin{array}{l}\text { Insulina glargina - } 100 \mathrm{Ui} / \mathrm{ml}-3 \mathrm{ml} \text { - } \\
\text { refil / unidade }\end{array}$ & Diabetes mellitus & Avaliado pela CONITEC. Decisão de não incorporar ao SUS publicada em 05/09/2014. \\
\hline $\begin{array}{l}\text { Insulina lispro - } 100 \mathrm{Ui} / \mathrm{ml} \mathrm{-} 3 \mathrm{ml} \text { - refil / } \\
\text { unidade }\end{array}$ & Diabetes mellitus & Avaliado pela CONITEC. Decisão de não incorporar ao SUS publicada em 05/09/2014. \\
\hline $\begin{array}{l}\text { Ranibizumabe - } 10 \mathrm{mg} / \mathrm{ml}-0,23 \mathrm{ml} / \\
\text { ampola }\end{array}$ & $\begin{array}{l}\text { Degeneração macular relacionada à } \\
\text { idade (DMRI), edema macular } \\
\text { diabético (EMD), edema macular } \\
\text { secundário à oclusão de veia da } \\
\text { retina (OVR) }\end{array}$ & $\begin{array}{l}\text { Avaliado pela CONITEC para DMRI. Decisão de não incorporar ao SUS publicada em } \\
\text { 10/04/2015. } \\
\text { Não avaliado pela CONITEC para EMD. } \\
\text { Não avaliado pela CONITEC para OVR. }\end{array}$ \\
\hline $\begin{array}{l}\text { Rivaroxabana - } 20 \mathrm{mg} / \text { comprimido } \\
\text { revestido }\end{array}$ & $\begin{array}{l}\text { Prevenção de acidente vascular } \\
\text { cerebral }\end{array}$ & Avaliado pela CONITEC. Decisão de não incorporar ao SUS publicada em 10/02/2016. \\
\hline
\end{tabular}

Fonte: Elaborado pelos autores. 
Observa-se que os 6 DRS com os IPJS mais elevados respondem por $41,6 \%$ dessas demandas, e por mais de metade das demandas de ranizumabe $(59,8 \%)$, clopidogrel $(60,8 \%)$ e rivaroxabana $(69,4 \%)$.

\section{Considerações finais}

Várias estratégias foram pensadas para lidar com as ações judiciais para obtenção de medicamentos por pacientes no estado de São Paulo. Poucas informações foram publicadas quanto ao funcionamento e resultados dessas estratégias, particularmente aquelas relativas ao sistema judiciário.

Em parceria com a CONITEC, a força de trabalho da REPATS e suas produções tem sido uma importante ferramenta tanto para responder às demandas judiciais, por meio da elaboração de notas técnicas junto à equipe do CODES, como para solicitar incorporações de novas tecnologias no SUS, através da elaboração de pareceres técnico-científicos e avaliações econômicas.

Embora os dados mostrem que continuou crescendo o número de ações judiciais mesmo após a adoção dessas estratégias, o sistema de regulação implantado pela SES$\mathrm{SP}$, em conjunto com as decisões da CONITEC para incorporação de alguns dos medicamentos judicializados, contribuiu parcialmente para a redução dos gastos (26).

Essas estratégias, no entanto, são consideradas ainda insuficientes. Atualmente a SES-SP responde a aproximadamente 47 mil condenações, com um gasto anual estimado de 1 bilhão de reais. Por isso, a Secretaria de Saúde decidiu realizar uma parceria com a Universidade de São Paulo para a emissão de pareceres sobre eficácia dos medicamentos, além de estreitar o diálogo com o sistema judiciário ${ }^{8}$ De outro lado, seria necessário compreender melhor a situação dos seis DRS com os IPJS mais elevados, para delinear estratégias específicas. Não se trata apenas de reduzir gastos, como também de evitar que pacientes sejam iludidos pelo consumo de medicamentos que não são capazes de trazer qualquer benefício à sua saúde, e eventualmente até de comprometê-la ainda mais.

Espera-se que outros estados adotem medidas similares de aproximação com os magistrados, assim como o fizeram Minas Gerais e a própria CONITEC, que têm elaborado 8 SP cria ofensiva para combater 'judicialização' da Saúde. Disponível em:
http://www.saude.sp.gov.br/ses/noticias/2016/abril/sp-cria-ofensiva-para-combater-judicializacao-da-saude 
notas $^{9}$ e fichas técnicas ${ }^{10}$ para subsidiar os processos judiciais relativos a medicamentos. Medidas similares em vários estados que enfrentam essa situação podem contribuir para aprimorar o processo em todo o país.

Ações junto aos médicos, que são os que iniciam o processo ao prescrever medicamentos cuja eficácia e segurança são duvidosos, exigem uma participação mais efetiva do Conselho Federal de Medicina ${ }^{11}$. Sobre o papel dos médicos na judicialização da saúde, Gadelha (27) argumenta:

As alegações médicas para justificar a prescrição fora de padronização institucional ou governamental são repetidas, por disseminadas, e tanto se baseiam no Código de Ética Médica, como em raciocínios técnicos que, muitas vezes, traem limitações e equívocos, ...

O direito à saúde não significa direito ao que se apresenta como discricionariedade médica ou acesso ao que está disponibilizado no mercado. A decisão judicial a partir de uma prescrição médica não deve considerar somente as justificativas científicas que a acompanham (que podem ser incompletas ou indevidamente interpretadas).

Isso porque, para uma boa prática médica e o uso racional de procedimentos e medicamentos, contam a sua seleção adequada, a melhor conduta para o caso em pauta e o porte tecnológico da instituição em que se dá o atendimento, bem como a observância às diretrizes e protocolos estabelecidos com base em rigorosa busca da literatura científica especializada e resultante de criteriosa análise técnico-científica, que considera todas as alternativas disponíveis, em termos de resultado, segurança e custo-efetividade.

\section{Referências}

1. Piola SF, Barros ED, Nogueira RP, Servo LM, Sá EB, Paiva AB. Vinte anos da Constituição de 1988: o que significaram para a saúde da população brasileira? Capítulo 3. p. 97-172. In IPEA - Instituto de Pesquisa Econômica Aplicada / Diretoria de Estudos e Políticas Sociais. Políticas sociais acompanhamento e análise. Vinte anos da Constituição Federal. N. 17, v.

\footnotetext{
${ }^{9}$ Conselho Nacional de Justiça. Fórum da Saúde. Disponível em: http://www.cnj.jus.br/sobre-o-cnj/presidencia/455-acoese-programas/programas-de-a-a-z/forum-da-saude/79016-nats-notas-tecnicas

10 Comissão Nacional de Incorporação de Tecnologias no SUS. Direito e Saúde. Fichas técnicas. Disponível em: http://conitec.gov.br/index.php/direito-e-saude

11 CNJ cobra comprometimento do CFM para a redução da judicialização da saúde. Disponível em: http://www.cnj.jus.br/noticias/cnj/83619-cnj-cobra-do-cfm-mais-compromisso-para-a-reducao-da-judicializacao-da-saude
} 
1. Brasília, 2009. 280 p. [acesso 30 outubro 2016]. Disponível em: http://www.ipea.gov.br/portal/images/stories/PDFs/livros/bps_completo_1.pdf

2. Marques SB, Dallari SG. Garantia do direito social à assistência farmacêutica no Estado de São Paulo. Rev Saúde Pública, São Paulo. 2007;41(1):101-7. [acesso 30 outubro 2016]. Disponível em: http://www.revistas.usp.br/rsp/article/view/32199.

3. Vieira FS, Zucchi P. Distorções causadas pelas ações judiciais à política de medicamentos no Brasil. Rev. Saúde Pública [Internet]. 2007;41(2):214-22. [acesso 30 outubro 2016]. Disponível em: http://www.scielo.br/pdf/rsp/v41n2/5587.pdf

4. Chieffi AL, Barata RB. Judicialização da política pública de assistência farmacêutica e eqüidade. Cad. Saúde Pública [Internet]. 2009;25(8):1839-49. [acesso 30 outubro 2016]. Disponível em: http://www.scielo.br/pdf/csp/v25n8/20.pdf

5. Chieffi AL, Barata RCB. Ações judiciais: estratégia da indústria farmacêutica para introdução de novos medicamentos. Rev. Saúde Pública [Internet]. 2010;44(3):42129. [acesso 30 outubro 2016]. Disponível em: http://www.scielo.br/pdf/rsp/v44n3/05.pdf

6. Naffah Filho M, Chieffi AC, Correa MC. S-Codes: um novo sistema de informações sobre ações judiciais da Secretaria de Estado da Saúde de São Paulo. Bepa. 2010;7(84):18-30. [acesso $\quad 30 \quad$ outubro 2016]. $\quad$ Disponível em: http://portal.saude.sp.gov.br/resources/ses/perfil/profissional-dasaude/destaques//boletim_bepa_84_gais_7.pdf

7. Yoshinaga JY. Judicialização do direito à saúde: a experiência do estado de São Paulo na adoção de estratégias judiciais e extra-judiciais para lidar com esta realidade. Revista Eletrônica sobre a reforma do estado. 2011;24:1-21. [acesso 30 outubro 2016]. Disponível

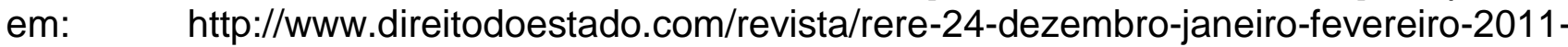
juliana-yumi.pdf

8. Chieffi AL, Siqueira PSF. Judicialização da saúde no estado de São Paulo. In Judicialização da saúde no Brasil / organizadoras: Lenir Santos, Fernanda Terrazas. Campinas, SP: Saberes Editora, 2014. p. 267-301.

9. Schulze CJ. O papel do Conselho Nacional de Justiça na judicialização da saúde. In Direito à Saúde, coleção Para Entender a Gestão do SUS. Conselho Nacional de Secretários de Saúde - CONASS, 2015. [acesso 30 outubro 2016]. Disponível em: http://www.conass.org.br/biblioteca/pdf/colecao2015/CONASS-DIREITO_A_SAUDEART_10B.pdf

10. Trindade EM, Martins PN, Zamberlan AGON, Opromolla PA, Toma TS, Muller SS, et al. Experiência da rede paulista de ATS na parceria com a CONITEC. Gestão \& Saúde. [periódico na internet]. 2015; 6(4):3297-312. [acesso 30 outubro 2016]. Disponível em: http://gestaoesaude.unb.br/index.php/gestaoesaude/article/view/1417/pdf

11. Trindade EM, Zamberlan AGON, Toma TS, Venancio SI, Müller SS, Opromolla PA. Rede Paulista de Avaliação de Tecnologias de Saúde: primeiros passos. BIS, Bol. Inst. Saúde 
(Impr.) [periódico na Internet]. 2013;14(2):151-8. [acesso em 26 out. 2016]. Disponível em: http://periodicos.ses.sp.bvs.br/pdf/bis/v14n2/v14n2a04.pdf

12. Secretaria de Estado da Saúde de São Paulo. Gabinete do Secretário. Resolução SS54, de 11-05-2012. Diário Oficial. Poder Executivo. Estado de São Paulo. Seção I, № $89-$ DOE de 12/05/12 - Seção 1 - p.37. [acesso em 30 novembro 2016]. Disponível em: http://www.saude.sp.gov.br/resources/ses/perfil/gestor/comissao-

farmacologia/resolucao_ss_54_de_11_de_maio_2012.pdf

13. Comissão Nacional de Incorporação de Tecnologias no SUS. Insulinas análogas de longa ação para Diabetes Mellitus tipo II. Relatório de Recomendação da Comissão Nacional de Incorporação de Tecnologias no SUS - CONITEC - 103, setembro de 2014. [acesso em 30 novembro 2016]. Disponível em: http://conitec.gov.br/images/Insulinastipo2-103FINAL.pdf

14. Comissão Nacional de Incorporação de Tecnologias no SUS. Insulinas análogas para Diabetes Mellitus tipo I. Relatório de Recomendação da Comissão Nacional de Incorporação de Tecnologias no SUS - CONITEC - 114, setembro de 2014. [acesso em 30 novembro 2016]. Disponível em: http://conitec.gov.br/images/Relatorios/2014/Insulinas-tipol-FINAL.pdf

15. Comissão Nacional de Incorporação de Tecnologias no SUS. Ranibizumabe para Degeneração Macular Relacionada à idade. Relatório de Recomendação da Comissão Nacional de Incorporação de Tecnologias no SUS - CONITEC - 119, abril de 2015. [acesso em 30 novembro 2016]. Disponível em: http://conitec.gov.br/images/Relatorios/2015/Relatorio_Ranibizumabe_DMRI_final.pdf

16. Comissão Nacional de Incorporação de Tecnologias no SUS. Ranibizumabe para o tratamento do edema macular diabético. Ficha técnica sobre medicamentos, junho de 2015. [acesso em 30 novembro 2016]. Disponível em: http://conitec.gov.br/images/FichasTecnicas/Ranibizumabe_EMD_jun2015.pdf

17. Comissão Nacional de Incorporação de Tecnologias no SUS. Ranibizumabe para o tratamento do edema macular secundário à oclusão de veia da retina. Ficha técnica sobre medicamentos, junho de 2015. [acesso em 30 novembro 2016]. Disponível em: http://conitec.gov.br/images/FichasTecnicas/Ranibizumabe_OVR_jun2015.pdf

18. Comissão Nacional de Incorporação de Tecnologias no SUS. Apixabana, rivoraxabana e dabigratana em pacientes com fibrilação atrial não valvar. Relatório de Recomendação da Comissão Nacional de Incorporação de Tecnologias no SUS - CONITEC - 195, fevereiro de 2016. [acesso em 30 novembro 2016]. Disponível em: http://conitec.gov.br/images/Relatorios/2016/Relatrio_Anticoagulantes_final.pdf

19. Comissão Nacional de Incorporação de Tecnologias no SUS. Hilano G-F 20 para o uso intra-articular no tratamento de dor associada com a osteoartrose do joelho. Relatório de Recomendação da Comissão Nacional de Incorporação de Tecnologias no SUS - CONITEC - 132, dezembro de 2014. [acesso em 30 novembro 2016]. Disponível em: http://conitec.gov.br/images/Artigos_Publicacoes/Relatorio_Hilano_Osteoartrite_FINAL.pdf 
20. Núcleo de Avaliação de Tecnologias em Saúde. Universidade Federal de Minas Gerais. NOTA TÉCNICA 88/2013. Uso de sulfato de glucosamina e sulfato de condroitina no tratamento da osteoartrite de joelhos. [acesso em 30 novembro 2016]. Disponível em: http://www.cnj.jus.br/files/conteudo/destaques/arquivo/2015/04/506f43106af30b29b956a0e 65e33ed55.pdf

21. Comissão Nacional de Incorporação de Tecnologias no SUS. Inibidores de Protease (Boceprevir e Telaprevir) para o tratamento da Hepatite Crônica C. Relatório de Recomendação da Comissão Nacional de Incorporação de Tecnologias no SUS - CONITEC - 01, julho de 2012. [acesso em 30 novembro 2016]. Disponível em: http://conitec.gov.br/images/Relatorios/2012/Boceprevir_Telaprevir_final.pdf

22. Comissão Nacional de Incorporação de Tecnologias no SUS. Telaprevir, boceprevir, filgrastim e alfaepoetina para o tratamento da hepatite C. Relatório de Recomendação da Comissão Nacional de Incorporação de Tecnologias no SUS - CONITEC - 222, maio de 2016. [acesso em 30 novembro 2016]. Disponível em: http://conitec.gov.br/images/Relatorios/2016/Relatorio_Telaprevir-Boceprevir-FilgrastimAlfaepoetina_Hepatite_Recomendacao.pdf

23. Comissão Nacional de Incorporação de Tecnologias no SUS. Cinacalcete para tratamento de pacientes com hiperparatireoidismo secundário à doença renal, em diálise e refratários à terapia convencional. Relatório de Recomendação da Comissão Nacional de Incorporação de Tecnologias no SUS - CONITEC - 763, outubro de 2013. [acesso em 30 novembro 2016]. Disponível em: http://conitec.gov.br/images/Incorporados/CinacalceteHPTS-FINAL.pdf

24. Comissão Nacional de Incorporação de Tecnologias no SUS. Cinacalcete e paricalcitol para o tratamento de pacientes com hiperparatireoidismo secundário à doença renal (HPTS), em diálise e refratários à terapia convencional. Relatório de Recomendação da Comissão Nacional de Incorporação de Tecnologias no SUS - CONITEC - 176, setembro de 2015. [acesso em 30 novembro 2016]. Disponível em: http://conitec.gov.br/images/Relatorios/2015/CinacalceteeParicalcitol_HPTS_final.pdf

25. Brasil. Ministério da Saúde. Secretaria de Ciência, Tecnologia e Insumos Estratégicos. Departamento de Assistência Farmacêutica e Insumos Estratégicos. Relação Nacional de Medicamentos Essenciais: RENAME 2014 / Ministério da Saúde, Secretaria de Ciência, Tecnologia e Insumos Estratégicos, Departamento de Assistência Farmacêutica e Insumos Estratégicos. - 9. ed. rev. e atual. - Brasília: Ministério da Saúde, 2015. 230 p. [acesso em 30 novembro 2016]. Disponível em: http://conitec.gov.br/images/Artigos_Publicacoes/Rename/Rename-2014-Julho2015.pdf

26. Silva EP. O papel dos comitês técnicos de especialistas na gestão de novas tecnologias em saúde. In Avaliação de Tecnologias de Saúde: desafios e propostas para a gestão / organizado por Tereza Setsuko Toma et al. São Paulo: Instituto de Saúde, 2015. P. 57-63. [acesso em 30 novembro 2016]. Disponível em: http://www.saude.sp.gov.br/resources/instituto-de-saude/homepage/temas-saudecoletiva/pdfs/ats_inova_saude_capa_miolo_errata.pdf 
Cuadernos Iberomericanos

de Derecho Sanitario

27. Gadelha MIP. O papel dos médicos na judicialização da Saúde. Revista CEJ, Brasília, Ano XVIII. 2014;62:65-70. [acesso em 30 novembro 2016]. Disponível em: http://www.cjf.jus.br/ojs2/index.php/revcej/article/viewFile/1862/1813

Submetido em: 23.1.2017 Aprovado em: 23.3.2017

Como citar este artigo:

Toma TS, Soares AC, Siqueira PSF, Domingues R. Estratégias para lidar com as ações judiciais de medicamentos no estado de São Paulo. Revista Cadernos Ibero-Americanos de Direito Sanitário. 2017 jan./mar, 6(1):35-54. 